\title{
PAX2: a reliable marker for nephrogenic adenoma
}

\author{
Guo-Xia Tong ${ }^{1}$, Jonathan Melamed ${ }^{2}$, Mahesh Mansukhani ${ }^{1}$, Lorenzo Memeo ${ }^{1}$, \\ Osvaldo Hernandez ${ }^{2}$, Fang-Ming Deng ${ }^{2}$, Luis Chiriboga ${ }^{2}$ and Jerry Waisman ${ }^{2}$ \\ ${ }^{1}$ Department of Pathology, College of Physicians and Surgeons, Columbia University, New York, NY, USA \\ and ${ }^{2}$ Department of Pathology, New York University Medical Center, New York, NY, USA
}

\begin{abstract}
Nephrogenic adenoma is a rare lesion of the urinary tract. The diagnosis usually is straightforward when characteristic microscopic and clinical findings are present, and the entity is familiar. However, misdiagnosis, in particular of adenocarcinoma of the prostate gland, may occur. Immunohistochemical stains often are needed to make such a distinction, but currently available markers offered only partial help. It recently was demonstrated that nephrogenic adenoma in renal transplant patients originated from the renal tubular epithelium. This newly proved, but long sought information may be helpful in the differential diagnosis of neophrogenic adenoma. In this study, we investigated the expression of a renal transcription factor, PAX2, in 39 nonrenal transplant-related nephrogenic adenomas, 100 adenocarcinomas of the prostate gland, and 47 urothelial carcinomas of the urinary tract. A strong and distinct nuclear staining of PAX2 was found in all 39 cases of nephrogenic adenoma (100\%), but not in normal prostate tissue, normal urothelium, adenocarcinomas of the prostate gland, and invasive urothelial carcinomas. Focal CD10 was detected in six of 13 nephrogenic adenomas in the superficial papillary component and in normal prostate epithelium, normal urothelium, lymphocytes, adenocarcinoma of the prostate gland, and urothelial carcinoma. There was no uroplakins detected in nephrogenic adenoma. Therefore, these findings are suggesting that nephrogenic adenoma in nonrenal transplant patients may also arise from the renal epithelium, as did the comparable lesions after transplantation. PAX2 is a specific and sensitive immunohistochemical marker in identification and differential diagnosis of nephrogenic adenoma.

Modern Pathology (2006) 19, 356-363. doi:10.1038/modpathol.3800535; published online 6 January 2006
\end{abstract}

Keywords: nephrogenic adenoma; immunohistochemistry; PAX2

Nephrogenic adenoma is a benign, tumor-like lesion of the urinary tract marked by a proliferation of cuboidal cells lining small tubules and cysts in the lamina propria with or without polypoid papillary projections into the lumen. Nephrogenic adenoma occurs in the urinary tract from the renal pelvis through the urethra with the most frequent location being the urinary bladder. ${ }^{1,2}$ Nephrogenic adenoma is often associated with other chronic conditions of the urinary tract, such as recurrent infections, calculi, congenital abnormalities, and previous trauma including operations, intravesical Bacillus Calmette-Guerin (BCG) treatment, radiation therapy, and renal transplantation. ${ }^{3-7}$ The microscopic findings in nephrogenic adenoma have been well

Correspondence: Dr G-X Tong, MD, PhD, Department of Pathology, College of Physicians and Surgeons, Columbia University, Room VC 14-238, 630 West, 168th Street, New York, NY 10032, USA.

E-mail: gt2125@columbia.edu

Received 27 July 2005; revised 20 October 2005; accepted 21 October 2005; published online 6 January 2006 described, and the diagnosis usually is straightforward. ${ }^{4-9}$ However, small tubules, lacking a basal cell layer and occasional cytological atypia may lead to the erroneous diagnosis of malignant tumors of the lower urinary tract, particularly adenocarcinoma of the prostate gland, clear cell carcinoma of the urinary bladder, and urothelial carcinoma. ${ }^{10-14}$ Immunohistochemical markers, such as CK7, CK20, 34betaE12, p53, P63, prostate-specific antigen (PSA), and prostate-specific alkaline phosphatase have been used with partial success to refine the diagnosis of nephrogenic adenoma. ${ }^{11,13,15,16}$

The histogenesis of nephrogenic adenoma long has been a matter of debate, but recently Mazal et $a l^{17}$ demonstrated that nephrogenic adenoma in renal transplant patients originates from the donor kidneys and is positive for renal-specific antigens. Therefore, nephrogenic adenoma may be considered a transplant of renal tubular epithelium in the urinary tract. ${ }^{17,18}$ These novel findings suggest a new way to identify nephrogenic adenoma and the differential diagnosis between other lesions. In this study, we investigated the expression of a 
renal-specific transcriptional factor, $\mathrm{PAX} 2$, in nonrenal transplant-related nephrogenic adenomas using immunohistochemistry, ${ }^{19}$ and the utility of PAX2 in the differentiation of nephrogenic adenomas from adenocarcinoma of the prostate gland and invasive urothelial carcinoma using high throughput tissue microarrays. Immunohistochemical stains for CD10, a renal tumor-related marker, and for uroplakins, the urothelium-specific proteins, were included in this study to address other contended lines of histogenesis, such as urothelium..$^{20,21}$

\section{Materials and methods}

A total of 39 nephrogenic adenomas from 36 patients were retrieved from the files of 2001-2005 (consecutive but excluded cases with insufficient material) at Columbia Presbyterian Hospital and New York University Medical Center Tisch Hospital. The diagnoses of nephrogenic adenoma were confirmed by reviewing all routine H\&E-stained slides. The specimens included 23 biopsies of the urinary bladder, distal urethra, or urethral diverticuli, 12 transurethral prostate resections, one urethral diverticulum, one partial cystectomy, one radical cystectomy, and one radical prostatectomy. Reactive hyperplasia was seen in the urothelium adjacent to nephrogenic adenoma in six cases. The cystitis cystica and cystitis glandularis were found to be coexistent with nephrogenic adenoma in three and two cases, respectively. Variable numbers of Brunn's nests were also present in five specimens. A series of unstained sections $(4 \mu \mathrm{m})$ obtained from the paraffin-embedded blocks of tissue were used for immunohistochemical studies.

The tissue microarrays of adenocarcinoma of prostate and normal prostate tissue were constructed with $0.6 \mathrm{~mm}$ cores from formalin-fixed, paraffin-embedded tissues of 100 chronologically consecutive radical prostatectomy specimen at New York University Medical Center Tisch hospital between 2001 and 2002 as described elsewhere. ${ }^{22}$ In brief, four cores $(0.6 \mathrm{~mm})$ from the cancer areas and one core of normal prostate tissue were procured from each case. This tissue microarray represented a cohort of 100 adenocarcinomas of the prostate and of normal prostate tissue, containing 400 cores for tumor and 100 cores of normal prostate tissue, all confirmed by H\&E stain. The cell culture pellets of prostate cancer cell lines (PC3 and LNCaP) and the pellets of short-term culture of normal prostate tissue were included as controls. Similarly, a tissue microarray of 47 invasive urothelial carcinoma was constructed with three cores of tumor from each case, included 39 urothelial carcinomas of common type, three micropapillary variant of urothelial carcinoma, two adenocarcinoma of the urinary bladder, two squamous cell carcinoma of the urinary bladder, and one urothelial carcinoma with focal clear cell change. The normal urothelium and prostate tissue in the nephrogenic adenoma specimen were used as internal controls.

Immunohistochemistry was performed using the avidin-biotin peroxidase method after antigen retrieval as described by Daniel et al. ${ }^{23}$ In brief, the sections were deparaffinized and incubated with $3 \%$ hydrogen peroxide for $15-20 \mathrm{~min}$ to block endogenous peroxidase activity. Antigen retrieval was accomplished by steaming the sections of paraffin-embedded tissues for $15 \mathrm{~min}$ in $10 \mathrm{mM}$ sodium citrate buffer, $\mathrm{pH}$ 6.0, in a microwave oven. The sections then were incubated sequentially with primary antibodies (overnight at room temperature for PAX2 and uroplakin antibodies), biotinylated secondary antibodies, avidin-biotin complex, and chromogenic substrate 3, 3'-DAB. The primary antibodies used in this study included polyclonal antibody against human PAX2 (Zymed), monoclonal antibody for CD10 (Dako), polyclonal antibodies for uroplakin-IA, -IB, -II, and a monoclonal antibody for uroplakin-III (kindly provided by Dr TT Sun, New York University Medical School, NY, USA).

\section{Results}

\section{Summary of Clinical Histories and Microscopic Features}

The 39 nephrogenic adenomas were from 36 patients (31 male and five female) with ages from 14 to 84 years (mean 65 years). In all, 21 nephrogenic adenomas were located in the urinary bladder, 16 in the prostate urethra, and two in the distal urethra. Associated conditions (in the order of frequency) included papillary urothelial carcinoma or carcinoma in situ $(n=14)$, benign prostate hyperplasia or vesical neck constriction $(n=10)$, chronic cystitis $(n=5)$, adenocarcinoma of the prostate $(n=4)$, partial or total nephrectomies $(n=3)$ for oncocytoma and urothelial carcinoma of the renal pelvis, urethral diverticuli or congenital urogenital malformations $(n=2)$. Nephrogenic adenoma was the primary abnormal finding and was detected in the first biopsies in 15 patients associated with benign conditions, including five patients with chronic inflammation, three with nephrectomy for oncocytoma and urothelial carcinoma of the renal pelvis, three with benign prostate hyperplasia, three with urethral strictures, and one with bladder diverticulum. The remainder of nephrogenic adenomas appeared after one to eight urologic procedures, including biopsies of bladder, transurethral resections of prostate, partial cystectomies, or suprapubic prostatectomies. These procedures were mainly for the diagnosis and follow-up of urothelial carcinoma and in most intravesical BCG or chemotherapy had been administered. Five nephrogenic adenomas occurred in a previous biopsy site after a 3 to 4 -month interval. For most cases, nephrogenic adenoma was an incidental microscopic finding except in two where cystoscopy 
revealed polypoid masses and papillary urothelial carcinoma was suspected. The follow-up information was obtained from available patient medical records in our files and result of subsequent biopsies. The follow-up period varied from 3 months to 5 years. Most of nephgrogenic adenomas remained unchanged in multiple subsequent biopsies during this period. One patient developed the rare nested variant of urothelial carcinoma 3 years after the diagnosis of nephrogenic adenoma.

In all, 32 nephrogenic adenomas had the characteristic triad of microscopic features that is variably small tubules, microcysts, and papillary projections all with a single layer of epithelial cells (Figure 1a and b). Papillary projections were the exclusive findings in four cases (Figure 1c). Two cases had only a few small tubules in the lamina propia of the urinary bladder or in the prostate stroma. Microcysts were frequently seen adjacent to typical lesions and were the dominant component in one prostate urethral nephrogenic adenoma (Figure 1d). The epithelial cells were cuboid or columnar in the tubules and the papillary projections, and flat in the microcysts. They were bland with a moderate amount of eosinophilic or granular cytoplasm and small regular round nuclei. Nucleoli were usually inconspicuous. In two cases, the small tubules contained blue-tinged mucinous secretions (Figure 4a) or crystalloids (Figure 4c), which simulated the acini of adenocarcinoma of the prostate gland. Mild cytologic atypia was seen in two cases showing nuclear pleomorphism and prominent nucleoli (Figure 4e).

\section{Immunohistochemistry Findings}

PAX2 is a member of 'paired box' family of transcription factors and is present in the nuclei of cells. ${ }^{19}$ PAX2 is found in the epithelial cells of fetal kidneys of all tubular segments and of Bowman's capsules (Figure 2). In adult kidneys, the level of PAX2 and number of positive cells are decreased significantly. There was no PAX2 detected in the
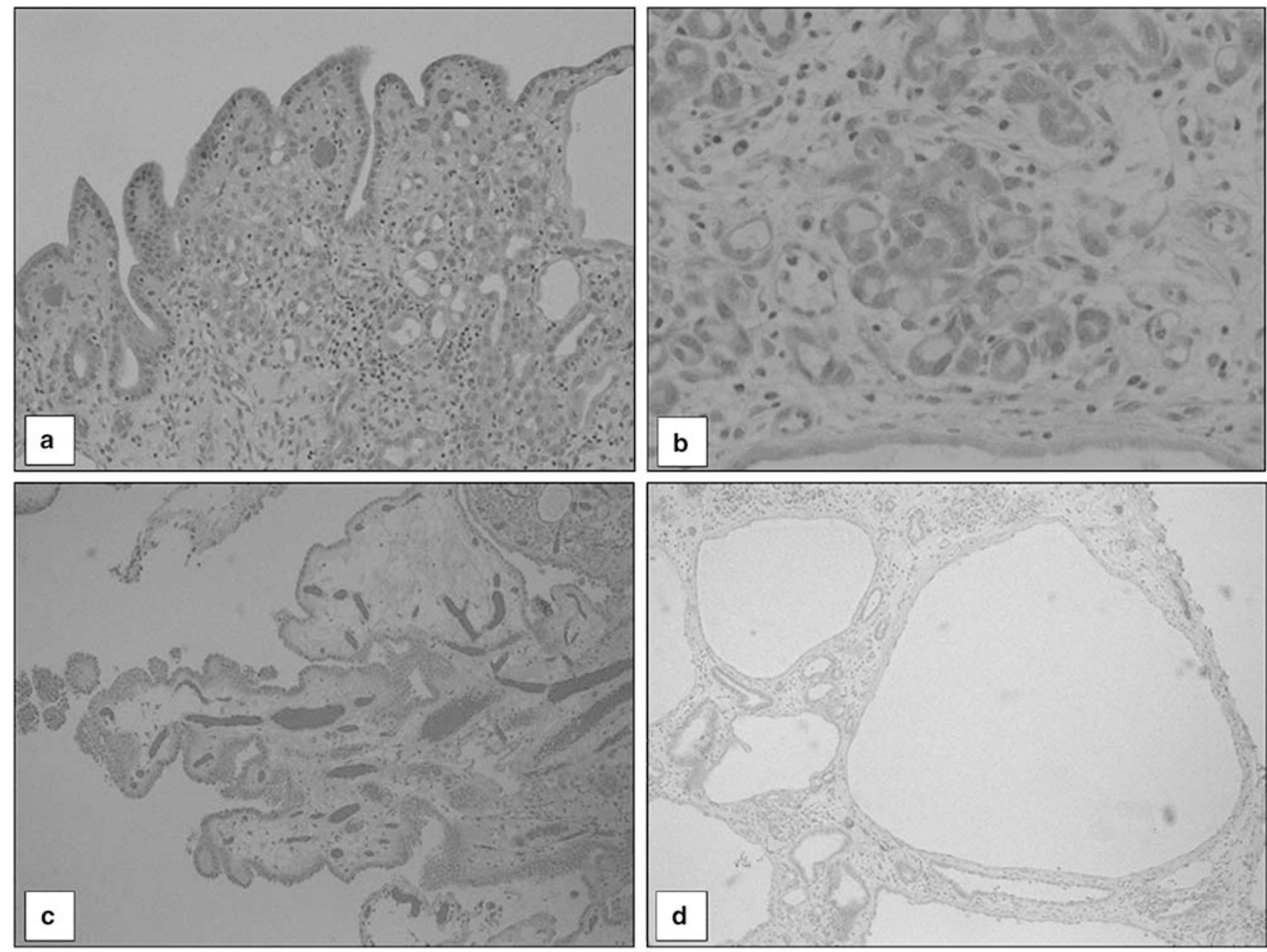

Figure 1 The histological features of nephrogenic adenoma. (a) Nephrogenic adenoma with characteristic triads of surface papillary projections, small tubules and microcysts in the lamina propria $(\mathrm{H} \& \mathrm{E}, \times 100)$. (b) Small tubules lined by a layer of bland epithelial cells in the lamina propria $(\mathrm{H} \& \mathrm{E}, \times 400)$. (c) Nephrogenic adenoma with prominent surface papillary structures $(\mathrm{H} \& \mathrm{E}, \times 100)$. (d) Nephrogenic adenoma with prominent microcysts $(\mathrm{H} \& \mathrm{E}, \times 100)$. 
normal lining of the renal pelvis, the ureter, the prostate gland, the urinary bladder, and the urethra. However, a strong and distinct staining of PAX2 was

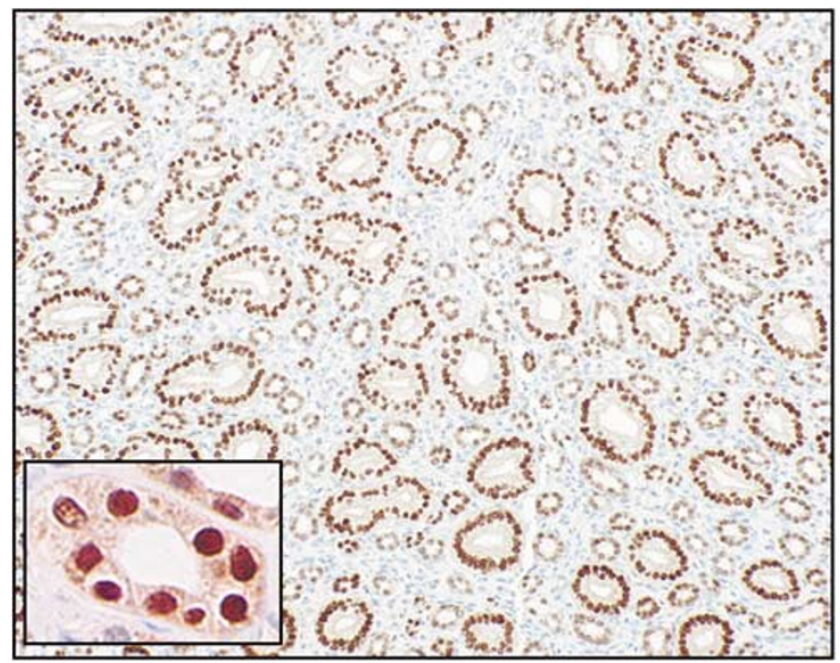

Figure 2 Expression of PAX2 in the fetal kidney. The PAX2 is distinctively localized in the nuclei of renal tubular epithelial cells (immunostain, $\times 100$; insert, $\times 400$ ).
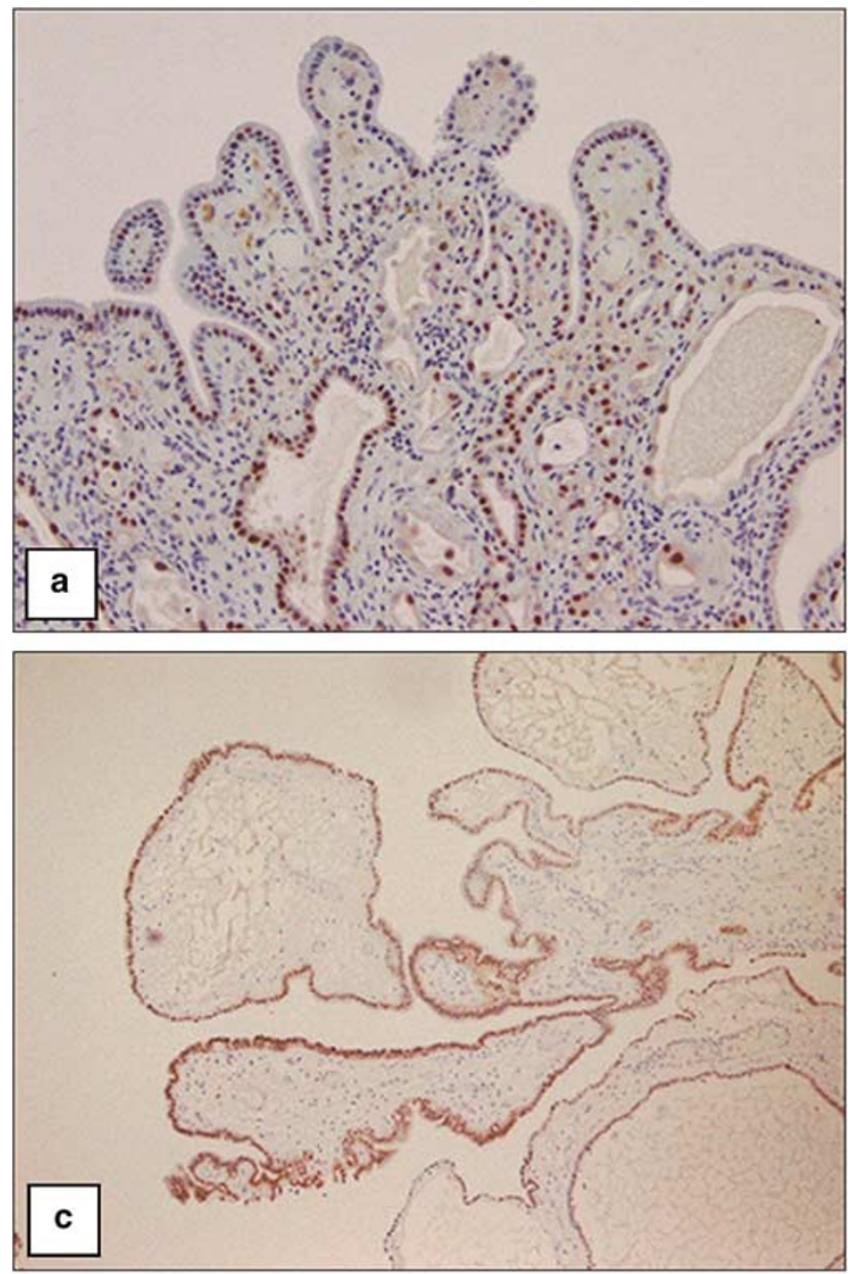

Figure 3 PAX2 is detected in all components of nephrogenic adenoma. (a) Nephrogenic adenoma with three characteristic components at lower magnification $(\times 100)$. (b) Small tubules $(\times 400)$. (c) Papillary fronds $(\times 100)$. (d) Microcysts $(\times 100)$.
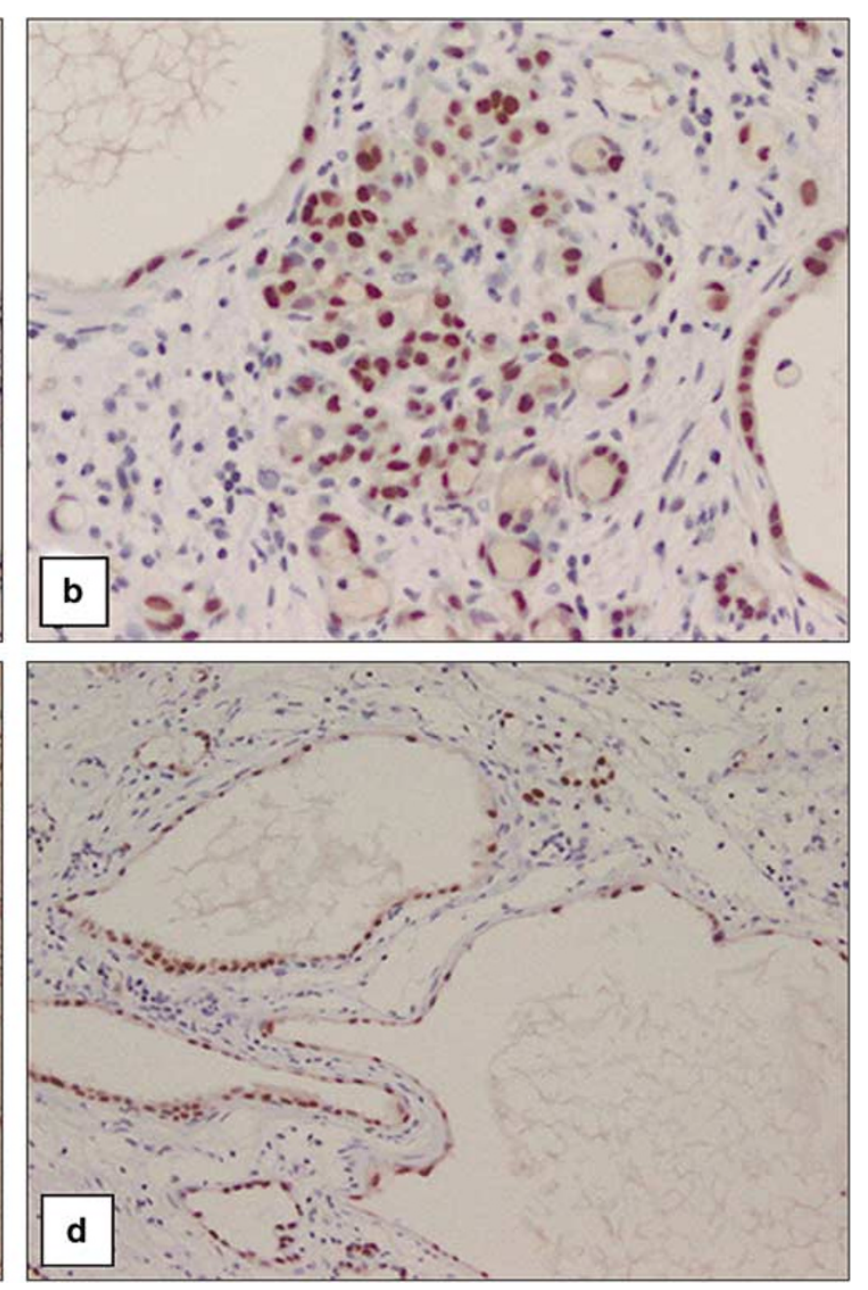

seen in the epithelial cells of all nephrogenic adenomas (39/39) and in each of its components (Figure 3a-d). Even small tubules with blue-tinged mucinous secretions, crystalloids, nuclear atypia or prominent nucleoli, were positive for PAX2 (Figure $4 \mathrm{~b}, \mathrm{~d}$, and f). There was no PAX2 expression in the 100 adenocarcinomas of the prostate gland or in the 47 invasive urothelial carcinomas in the tissue microarrays, and six papillary urothelial carcinomas coexistent with nephrogenic adenoma, nor was it found in the granulation tissue and granulomas subsequent to intravesical BCG, chemotherapy, or radiation. The coexisting cystitis cystica, cystitis glandularis, and Brunn's glands were also negative for PAX2. The only other PAX2-positive cells in the urinary bladder and prostate gland were isolated lymphocytes in the lymphoid aggregates associated with chronic cystitis, all readily distinguished from nephrogenic adenoma. Four types of antibodies to uroplakin-IA, -IB, -II, and -III were used in this study, and normally are expressed in the superficial cells of the urothelium. None of uroplakins were detected in these nephrogenic adenomas (0/39). Expression of CD10 was studied in 13 nephrogenic

Modern Pathology (2006) 19, 356-363 

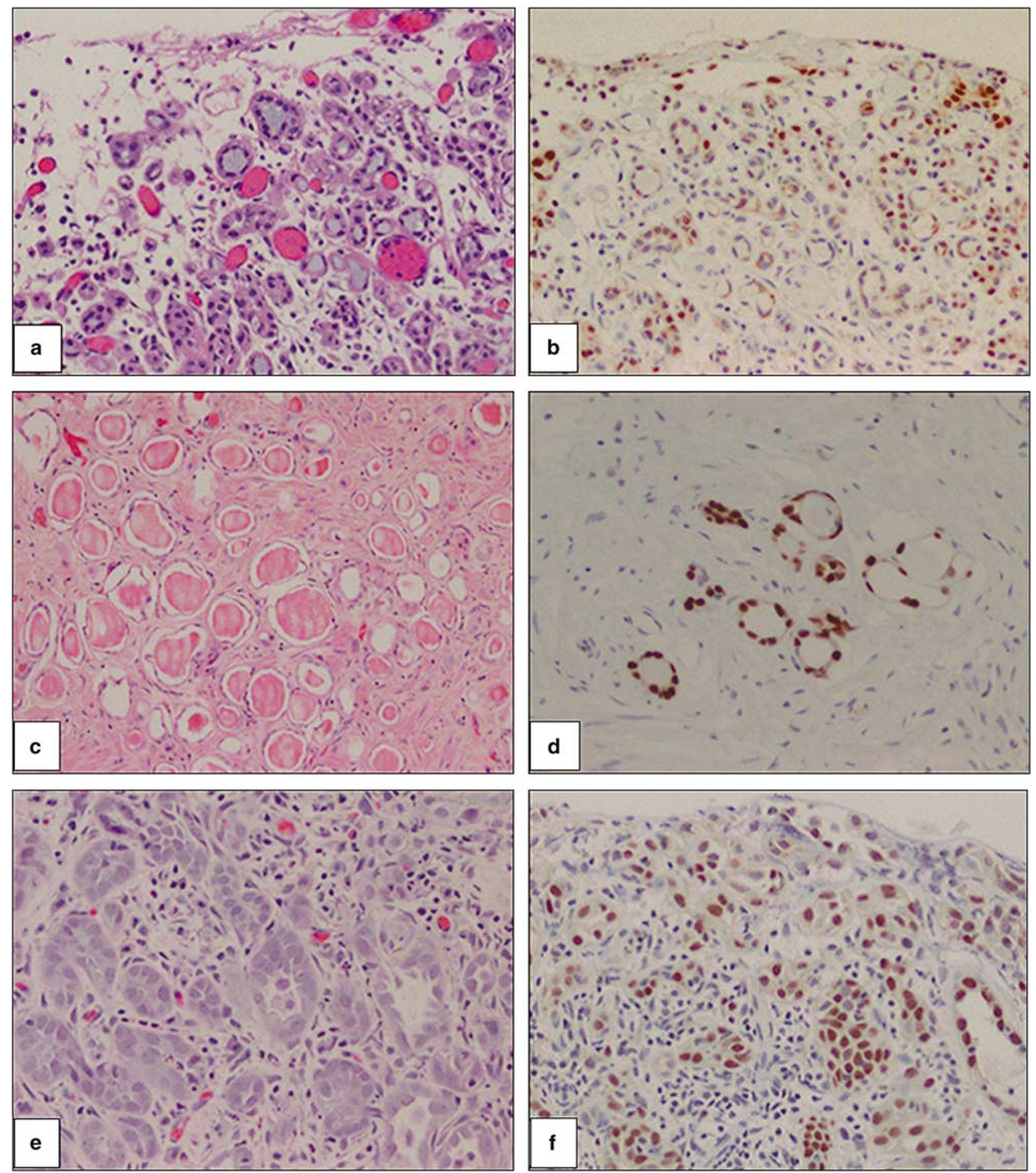

Figure 4 Expression of PAX2 in nephrogenic adenoma with atypical features. (a and b) Nephrogenic adenoma with small tubules with blue-tingled mucinous secretions (H\&E, PAX2 immunostain, respectively, $\times 200$ ). (c and d) Nephrogenic adenoma with small tubules or microcysts with crystalloids (H\&E, PAX2 immunostain, respectively, $\times 200$ ). (e and f) Nephrogenic adenoma with mild cytological atypia (H\&E, PAX2 immunostain, respectively, × 200).

adenomas. Focal CD10 was detected in six of 13 nephrogenic adenomas, mainly in the superficial papillary component. In addition, CD10 was found in normal prostate epithelia, normal urothelium, lymphocytes, adenocarcinoma of the prostate, and urothelial carcinoma. As expected, nephrogenic adenomas were positive for CK7 $(13 / 13)$ and Cam $5.2(13 / 13)$, and negative for CK20 (0/13). 


\section{Discussion}

Nephrogenic adenoma was described first by Davis in 1949 and later by Friedman and Kuhlenbech in $1950 .^{3}$ It has been reported throughout the entire urinary tract from the renal pelvis through the urethra. The most frequent location is the urinary bladder, particularly the trigone area. ${ }^{3,4}$ The histogenesis of nephrogenic adenoma has been a matter of debate, and various terms have been applied to the lesion, including adenomatous metaplasia, hamartoma, adenosis, and nephrogenic metaplasia, reflecting the different opinions on histogenesis. ${ }^{2,18}$ The prevalent one was that nephrogenic adenoma was a metaplastic change of de novo urothelium in response to persistent irritation, often associated with recurrent infection, calculi, anatomic defects, trauma, surgery, benign or malignant tumors of the lower urinary tract, and intravesicular therapies such as BCG or chemotherapeutic agents. ${ }^{3,4,24-26}$ However, there is no consistent evidence to support this commonly accepted theory.

Bhagavan demonstrated in 1991 by electron microscopy that the tubules of nephrogenic adenoma had ultrastructural features of renal proximal tubular cells and were negative for Tamm-Horsfall protein, a mucoprotein of the urothelium. ${ }^{27}$ This was the first indication that nephrogenic adenoma showed renal differentiation but was not followed by other investigation. However, patients with renal transplants have a high incidence of nephrogenic adenoma. ${ }^{5,6}$ Mazal et a ${ }^{17}$ studied the development of nephrogenic adenoma in 29 renal transplant patients in a period of 6-120 months after the transplantation. Of the 29 patients, 14 received kidneys from opposite-gender donors, which provided a unique cohort to study the possible renal origin of nephrogenic adenoma by detecting the sex chromosome using the fluorescent in situ hybridization technique. The Y-chromosome was detected in nephrogenic adenoma of female patients, who received kidneys from male donors. On the other hand, only X-chromosome but not Ychromosome was identified in nephrogenic adenoma of male patients who received female kidneys. Nephrogenic adenomas in these patients were positive for the proteins specific for renal tubular epithelial cells, including PAX2, aquaporin-1, peanut agglutinin, and Lotus tetragonolobus agglutinin. These results indicated that nephrogenic adenoma in renal transplant patients originated from the newly transplanted kidneys. Therefore, nephrogenic adenoma is not a metaplastic change of urothelium, but a migration and proliferation of dislodged renal tubular cells into the lower urinary tract. ${ }^{17,18}$ Increased shedding of renal tubular cells was the essential factor for high frequency of nephrogenic adenoma after transplantation. Immunosuppression had a minimal role since partial or total nephrectomy for renal tumors was noted in our study to be a contributing factor for nephrogenic adenoma development in nonrenal transplant patients.
As a member of 'paired box family,' PAX2 is a key transcription factor for nephric differentiation and expressed in the early embryonic stage of renal development. ${ }^{19,28,29}$ The level of PAX2 is higher in the tubular epithelial cells of fetal kidneys and is diminished or absent in adult renal tissue. ${ }^{30}$ Our study demonstrated this, too. PAX2 expression was enhanced in some pathological conditions, such as the multicystic dysplastic kidney, renal cell carcinoma, and Wilms' tumor. ${ }^{31}$ PAX2 was used in our study in part because the distinct nuclear staining facilitates interpretation of immunohistochemical stains. As a renal lineage determinant transcriptional factor, PAX2 may be more sensitive and specific in determining lesions of renal origin or with renal differentiation as some transcriptional factors in other systems, such as TTF- $1,{ }^{32}$ myogenin and MyoD1. ${ }^{33}$ Unlike the series of Mazal et al, ${ }^{17}$ we did not include renal transplant patients; but patients with chronic cystitis, vesical or urethral diverticula, benign prostate hyperplasia, nephrectomy, adenocarcinoma of the prostate gland, and papillary or invasive urothelial carcinoma. PAX2 is present unequivocally in the epithelial cells of all nephrogenic adenomas (39/39) in our study. Thus, we find a higher rate of staining than the originally reported $78 \%$ (33/42) by Mazal et al. ${ }^{17}$ The nephrogenic adenoma in conditions other than renal transplants expressed the renal lineage transcription factor and likely is derived from renal tubular cells as observed in the lesions in renal transplant patients.

Uroplakins are a group of newly identified, and structurally related proteins specific to urothelium and include four closely related proteins, uroplakinIA, -IB, -II, and -III. ${ }^{34-36}$ Uroplakins are present in the asymmetric unit membrane of the urothelium and are the most specific markers for urothelial differentiation and tumors of urothelial origin. ${ }^{21} \mathrm{We}$ reported that uroplakin-IA, -IB, and -III were detected in some nephrogenic adenomas in 2003 USCAP meeting in Washington. ${ }^{37}$ In subsequent study, we were not able to reproduce the initial findings. Instead, uroplakin was not detected in any NA in this study with antibodies against four major urothelium-specific proteins. We conclude that the discrepancy was due to the nonspecific staining of the antibodies used in the previous study. We like to take this opportunity to correct our initial statement. ${ }^{37}$ The current finding of lacking of uroplakin expression support that nephrogenic adenoma is not an urothelial lesion.

The microscopic features of nephrogenic adenoma seen are well known, and several patterns are recognized. $^{2,4,8}$ The diagnostic triad includes the presence of small tubules and microcysts in the lamina propria plus papillary projections on the surface, all lined by a single layer of flat, cuboid, or low columnar or 'hob-nail' cells with scant eosinophilic or amphophilic cytoplasm and uniform bland nuclei. The diagnosis of nephrogenic adenoma 
usually is straightforward; however, nephrogenic adenoma can be misdiagnosed as adenocarcinoma. In the prostate urethra it may be mistaken for adenocarcinoma of the prostate gland because the characteristic tubules lack basal cells. In addition, the tubules may extend into the underlying prostate stroma and appear to be infiltrative. Sometimes, the tubules may be lined with cells with prominent nucleoli or have blue mucinous material in the lumen. Immunohistochemical markers are helpful in the differential diagnosis with adenocarcinoma of the prostate gland..$^{10,38-41}$ Diffuse and intense cytoplasmic staining of PSA and prostate-specific alkaline phosphatase and lack of 34betaE12 staining are diagnostic of adenocarcinoma of the prostate gland. Cytoplasmic staining of CK7 and 34betaE12 and the absence of staining for PSA is suggestive of nephrogenic adenoma. However, only $11 \%$ of nephrogenic adenomas show diffuse immunoreactivity for 34betaE12, and whereas the remainder shows only focal reactivity $(44 \%)$ or no reactivity $(44 \%) .{ }^{10}$ Although nephrogenic adenoma was negative for PSA and prostate-specific alkaline phosphatase in initial reports, ${ }^{38,41}$ both were detected in nephrogenic adenomas when the antigen retrieval technique was applied. Thus, as many as 36 and $50 \%$ of nephrogenic adenoma were focally positive for PSA and prostate-specific alkaline phosphatase, respectively, using antigen retrieval. ${ }^{10}$ Alpha-methylacyl-CoA racemase (AMACR, P504S), a protein considered relatively specific for adenocarcinoma of the prostate gland is detected in nephrogenic adenoma of the urinary bladder and nephrogenic adenoma involving the prostate urethra and underlying stroma. ${ }^{41,42}$ These findings have limited the value of commonly used immunohistochemical markers in the differential diagnosis of nephrogenic adenoma. The nonreactivity of PAX2 staining in adenocarcinoma of the prostate gland and normal prostate epithelia in contrast to the uniformly positive staining in nephrogenic adenoma suggests that PAX2 is a specific and sensitive marker in the differentiation between these two lesions.

The common acute lymphoblastic leukemia antigen, CD10 is also expressed in renal epithelial cells. It is identified in renal neoplasm ${ }^{20}$ and nephrogenic adenoma. ${ }^{43}$ However, CD10 is less valuable than PAX2 in the diagnosis of nephrogenic adenoma because it was only found focally in some nephrogenic adenomas in our study. It is also present in the inflammatory cells and may obscure the differential diagnosis.

NA is less likely to be mistaken for conventional papillary or invasive urothelial carcinoma, but it may be confused with the rare clear cell carcinoma of the urinary bladder or the urethra. ${ }^{11-14}$ The most common pattern of clear cell carcinoma is tubulocystic formation, in which the tubules and cysts were lined by cuboid or columnar, flat or 'hobnail' epithelial cells with clear to eosinophilic cytoplasm. The epithelial cells of nephrogenic adenoma may have clear cytoplasm. Nephrogenic adenoma may be confused with clear cell carcinoma of the urinary bladder or the urethra, particularly in small superficial biopsies. PAX2 is not detected in normal urothelium, or in 47 invasive urothelial carcinomas including micropapillary variant, adenocarcinoma, squamous cell carcinoma, and one urothelial carcinoma with clear cell differentiation. Because of its rarity, we had no clear cell carcinoma of the urinary tract in our archival material. The expression of PAX2 and the significance of differential diagnosis in these rare tumors need to be investigated. A rightful diagnosis could be reached by the recognition of some histological features, such as significant nuclear atypia, prominent mitotic figures, foci of necrosis, and vascular or muscular invasion, and might be helped by immunohistochemical stains of MIB1 and p53.

In summary, the recent determination of histogenesis of nephrogenic adenoma as renal tubular epithelial origin provides a diagnostic tool for surgical pathologists. It allows using renal-specific markers, such as PAX2, to identify nephrogenic adenoma and differentiate it from other common entities with which it may be confused.

\section{References}

1 Kaver I, Merimsky E, Greenstein A, et al. Nephrogenic adenoma: benign proliferative lesion of urothelium. Urology 1985;26:506-508.

2 Ford TF, Watson GM, Cameron KM. Adenomatous metaplasia (nephrogenic adenoma) of urothelium. An analysis of 70 cases. Br J Urol 1985;57:427-433.

3 McIntire TL, Soloway MS, Murphy WM. Nephrogenic adenoma. Urology 1987;29:237-241.

4 Oliva E, Young RH. Nephrogenic adenoma of the urinary tract: a review of the microscopic appearance of 80 cases with emphasis on unusual features. Mod Pathol 1995;8:722-730.

5 Banyai-Falger S, Maier U, Susani M, et al. High incidence of nephrogenic adenoma of the bladder after renal transplantation. Transplantation 1998;65: 511-514.

6 Pycha A, Mian C, Reiter WJ, et al. Nephrogenic adenoma in renal transplant recipients: a truly benign lesion? Urology 1998;52:756-761.

7 Tse V, Khadra M, Eisinger D, et al. Nephrogenic adenoma of the bladder in renal transplant and nonrenal transplant patients: a review of 22 cases. Urology 1997;50:690-696.

8 Young RH. Pseudoneoplastic lesions of the urinary bladder and urethra: a selective review with emphasis on recent information. Semin Diagn Pathol 1997;14: 133-146.

9 Tamboli P, Ro JY, Amin MB, et al. Benign tumors and tumor-like lesions of the adult kidney. Part II: benign mesenchymal and mixed neoplasms, and tumor-like lesions. Adv Anat Pathol 2000;7:47-66.

10 Allan CH, Epstein JI. Nephrogenic adenoma of the prostatic urethra: a mimicker of prostate adenocarcinoma. Am J Surg Pathol 2001;25:802-808. 
11 Alsanjari N, Lynch MJ, Fisher C, et al. Vesical clear cell adenocarcinoma. V. Nephrogenic adenoma: a diagnostic problem. Histopathology 1995;27:43-49.

12 Oliva E, Amin MB, Jimenez R, et al. Clear cell carcinoma of the urinary bladder: a report and comparison of four tumors of mullerian origin and nine of probable urothelial origin with discussion of histogenesis and diagnostic problems. Am J Surg Pathol 2002;26:190-197.

13 Gilcrease MZ, Delgado R, Vuitch F, et al. Clear cell adenocarcinoma and nephrogenic adenoma of the urethra and urinary bladder: a histopathologic and immunohistochemical comparison. Hum Pathol 1998;29:1451-1456.

14 Oliva E, Young RH. Clear cell adenocarcinoma of the urethra: a clinicopathologic analysis of 19 cases. Mod Pathol 1996;9:513-520.

15 Gokaslan ST, Krueger JE, Albores-Saavedra J. Symptomatic nephrogenic metaplasia of ureter: a morphologic and immunohistochemical study of four cases. Mod Pathol 2002;15:765-770.

16 Muir TE, Cheville JC, Lager DJ. Metanephric adenoma, nephrogenic rests, and Wilms' tumor: a histologic and immunophenotypic comparison. Am J Surg Pathol 2001;25:1290-1296.

17 Mazal PR, Schaufler R, Altenhuber-Muller R, et al. Derivation of nephrogenic adenomas from renal tubular cells in kidney-transplant recipients. N Engl J Med 2002;347:653-659.

18 Ingelfinger JR. Nephrogenic adenomas as renal tubular outposts. N Engl J Med 2002;347:684-686.

19 Mansouri A, Hallonet M, Gruss P. Pax genes and their roles in cell differentiation and development. Curr Opin Cell Biol 1996;8:851-857.

20 Avery AK, Beckstead J, Renshaw AA, et al. Use of antibodies to RCC and CD10 in the differential diagnosis of renal neoplasms. Am J Surg Pathol 2000;24:203-210.

21 Sun TT, Liang FX, Wu XR. Uroplakins as markers of urothelial differentiation. Adv Exp Med Biol 1999;462: 7-18.

22 Melamed J, Datta MW, Becich MJ, et al. The cooperative prostate cancer tissue resource: a specimen and data resource for cancer researchers. Clin Cancer Res 2004;10:4614-4621.

23 Daniel L, Lechevallier E, Giorgi R, et al. Pax-2 expression in adult renal tumors. Hum Pathol 2001;32: 282-287.

24 Peeker R, Aldenborg F, Fall M. Nephrogenic adenoma-a study with special reference to clinical presentation. Br J Urol 1997;80:539-542.

25 O'Shea PA, Callaghan JF, Lawlor JB, et al. 'Nephrogenic adenoma': an unusual metaplastic change of urothelium. J Urol 1981;125:249-252.

26 Odze R, Begin LR. Tubular adenomatous metaplasia (nephrogenic adenoma) of the female urethra. Int J Gynecol Pathol 1989;8:374-380.

27 Bhagavan BS, Tiamson EM, Wenk RE, et al. Nephrogenc Adenoma of the urinary bladder and urethra. Hum Pathol 1991;12:907-916.
28 Bouchard M, Pfeffer P, Busslinger M. Functional equivalence of the transcription factors Pax2 and Pax5 in mouse development. Development 2000;127: 3703-3713.

29 Bouchard M, Souabni A, Mandler M, et al. Nephric lineage specification by Pax2 and Pax8. Genes Dev 2002;16:2958-2970.

30 Dahl E, Koseki H, Balling R. Pax genes and organogenesis. Bioessays 1997;19:755-756.

31 Winyard PJ, Risdon RA, Sams VR, et al. The PAX2 transcription factor is expressed in cystic and hyperproliferative dysplastic epithelia in human kidney malformations. J Clin Invest 1996;98:451-459.

32 Yatabe Y, Mitsudomi T, Takahashi T. TTF-1 expression in pulmonary adenocarcinomas. Am J Surg Pathol 2002;26:767-773.

33 Cessna $\mathrm{MH}$, Zhou H, Perkins SL, et al. Are myogenin and myoD1 expression specific for rhabdomyosarcoma? A study of 150 cases, with emphasis on spindle cell mimics. Am J Surg Pathol 2001;25:1150-1157.

$34 \mathrm{Wu}$ XR, Manabe M, Yu J, et al. Large scale purification and immunolocalization of bovine uroplakins II, III, and I. Molecular markers of urothelial differentiation. J Biol Chem 1990;265:19170-19179.

$35 \mathrm{Yu}$ J, Manabe M, Wu XR, et al. Uroplakin I: a 27-kD protein associated with the asymmetric unit membrane of mammalian urothelium. J Cell Biol 1990;111: 1207-1216.

$36 \mathrm{Yu}$ J, Wu XR, Sun TT. Uroplakins Ia and Ib, two major differentiation products of bladder epithelium, belong to a family of four transmembrane domain (4TM) proteins. J Biol Chem 2001;276:9924-9999.

37 Tong GX, Melamed J, Levine P, et al. Expression of uroplakins in nephrogenic adenoma: immunohistochemical evidence supporting urothelial origin. Mod Pathol 2003;16:173A.

38 Cheng L, Cheville JC, Sebo TJ, et al. Atypical nephrogenic metaplasia of the urinary tract: a precursor lesion? Cancer 2000;88:853-861.

39 Genega EM, Hutchinson B, Reuter VE, et al. Immunophenotype of high-grade prostatic adenocarcinoma and urothelial carcinoma. Mod Pathol 2000;13: 1186-1191.

40 Malpica A, Ro JY, Troncoso P, et al. Nephrogenic adenoma of the prostatic urethra involving the prostate gland: a clinicopathologic and immunohistochemical study of eight cases. Hum Pathol 1994;25:390-395.

41 Skinnider BF, Oliva E, Young RH, et al. Expression of alpha-methylacyl-CoA racemase (P504S) in nephrogenic adenoma: a significant immunohistochemical pitfall compounding the differential diagnosis with prostatic adenocarcinoma. Am J Surg Pathol 2004;28: 701-705.

42 Gupta A, Wang HL, Policarpio-Nicolas ML, et al. Expression of alpha-methylacyl-coenzyme a racemase in nephrogenic adenoma. Am J Surg Pathol 2004;28: 1224-1229.

43 Mai KT, Carnat T, Park W, et al. Mixed nephrogenic adenoma and Mullerian metaplasia of the urinary bladder mucosa. Pathology 2004;36:281-283. 